\title{
THE CONVERGENCE DETERMINING CLASS OF REGULAR OPEN SETS
}

\author{
LOTHAR ROGGE
}

\begin{abstract}
The purpose of this paper is to prove that every sequence of closed approximable measures defined on the Borelfield of a normal topological space with values in an abelian topological group is Cauchy convergent for all Borel sets if it is Cauchy convergent for all regular open sets. In particular every sequence of measures on the Borel-field of a perfectly normal topological space which is Cauchy convergent for all regular open sets is Cauchy convergent for all Borel sets, too.
\end{abstract}

1. Preliminaries. In this paper a topological group $G$ is always assumed to be abelian. The system of neighborhoods of the zero element of $G$ is denoted by $\mathscr{F}(0)$. A sequence $a_{n} \in G, n \in N$, is Cauchy convergent iff it is Cauchy convergent with respect to the uniformity:

$$
\{\{(a, b) \in G \times G: a-b \in F\}: F \in \mathscr{F}(0)\} .
$$

Let $(X, \mathscr{T})$ be a topological space. The closure of a set $A \subset X$ be denoted by $A^{c}$, and its interior by int $A$. A set $A \subset X$ is regular open iff $A=\operatorname{int} A^{c}$. The system of regular open sets is denoted by $\mathscr{T}_{r}$. The function $T \in \mathscr{T} \rightarrow T^{*}:=$ int $T^{c} \in \mathscr{T}_{r}$ has the following properties:

(i) $T^{* *}=T^{*}$;

(ii) $T \subset U$ implies $T^{*} \subset U^{*}$;

(iii) $T \cap U=\varnothing$ implies $T^{*} \cap U^{*}=\varnothing$;

(iv) $T^{c} \cap U^{c}=\varnothing$ implies $(T \cup U)^{*}=T^{*} \cup U^{*}$.

Let $\mathscr{B}$ be a $\sigma$-field on $X$ and $G$ be a topological group. A function $\mu: \mathscr{B} \rightarrow G$ is a measure iff for all disjoint sets $A_{i} \in \mathscr{B}, i \in N$, the sequence $\left(\sum_{i=1}^{n} \mu\left(A_{i}\right)\right)_{n \in N}$ converges to $\mu\left(\bigcup_{i \in N} A_{i}\right)$. Let $\mathscr{K} \subset \mathscr{B} ;$ a measure $\mu: \mathscr{B} \rightarrow G$ is $\mathscr{K}$-regular iff for each $A \in \mathscr{B}$ and each $F \in \mathscr{F}(0)$ there exists $K \in \mathscr{K}, K \subset A$ such that

$$
\mu(\mathscr{B} \cap(A-K)):=\{\mu(B \cap(A-K)): B \in \mathscr{B}\} \subset F .
$$

For the case of a Banach space $G$, regularity in the sense of this definition is the usual regularity, defined in terms of the semivariation.

Received by the editors May 3, 1971 and, in revised form, May 23, 1972. AMS (MOS) subject classifications (1970). Primary 60B10; Secondary 28A45.

Key words and phrases. Convergence of measures, regular open sets, topological group. 
If $\mathscr{K}$ is the system of compact or closed sets of a topological space then we speak of compact or closed approximable measures instead of $\mathscr{K}$ regular measures. Let $\mathfrak{M}$ be a family of measures defined on $\mathscr{B}$ with values in $G$ and $\mathscr{C}$ be a subsystem of $\mathscr{B} . \mathscr{C}$ is a convergence determining class for $\mathfrak{M}$ iff each sequence $\mu_{n} \in \mathfrak{M}, n \in N$, which is Cauchy convergent on $\mathscr{C}$, is Cauchy convergent on $\mathscr{B}$.

2. The main results. For a compact space $(X, \mathscr{T})$, Wells [6] proved that the system of regular open sets is a convergence determining class for the family of compact approximable real valued measures. Gänssler generalized this result in [1] to normal Hausdorff spaces and in [2] to regular Hausdorff spaces. For a normal space the following result is more general insofar as the measures are closed approximable only and not compact approximable any more. We remark that each measure on the Borel-field of a metric space is closed approximable whereas compact approximability of measures is only known for complete separable metric spaces. Furthermore the measures may assume values in a topological group instead of the real numbers. The proof seems, to the author, more transparent than the proofs of Wells [6] and Gänssler ([1], [2]) because the author directly uses the regular open sets whereas Wells and Gänssler describe the regular open sets by a complicated system of conditions. We remark that for a regular space Lemma 5 together with Theorem 3.11 of Gänssler [1] yields Gänssler's result in [2].

THEOREM 1. Let $(X, \mathscr{T})$ be a normal topological space. If a sequence of closed approximable measures on the Borel-field of $X$ with values in $a$ topological group is Cauchy convergent for the regular open sets, then it is Cauchy convergent for the Borel sets, too.

Proof. Let $\mathscr{K}$ be the system of closed sets and $\mu_{n}, n \in N$, be a sequence of $\mathscr{K}$-regular measures which is Cauchy convergent on $\mathscr{T}_{r}$. As $(X, \mathscr{T})$ is a normal topological space, for each $K \in \mathscr{K}, T \in \mathscr{T}$ with $K \subset T$, there exists $U \in \mathscr{T}_{r}$ such that $K \subset U \subset U^{c} \subset T$. Hence we may apply Lemma 5 , whence Lemma 7 yields that $\mathscr{T}$ and therefore $\mathscr{T}_{r}$ uniformly approximates $\mathscr{K}$ from above with respect to $\mu_{n}, n \in N$. Hence Cauchy convergence on $\mathscr{T}_{r}$ implies Cauchy convergence for all closed sets and therefore Cauchy convergence on $\mathscr{T}$. By Corollary 7 of [5], a sequence of closed approximable measures on a normal topological space is Cauchy convergent for all Borel sets if it is Cauchy convergent for all open sets. This implies the assertion.

We remark that every sequence of $\mathscr{K}$-regular measures with values in a complete topological group, which is Cauchy convergent for all Borel sets, converges for all Borel sets, and each limit function is a $\mathscr{K}$-regular measure [4, Theorem 8(2)]. 
If the measures are not closed approximable, Example 6 in [5] shows that even for a compact Hausdorff space Cauchy convergence for all open sets does not imply Cauchy convergence for all Borel sets. Using the regular open instead of the open sets we shall give a less complicated example. We construct two measures $v_{1}, v_{2}$ on the Borel-field of a compact Hausdorff space which coincide for the regular open but not for the open sets. Then the sequence of measures $\mu_{n}:=v_{1}$ or $\nu_{2}$ iff $n$ is even or odd converges for all regular open sets but not for all open sets.

EXAMPLE 2. Let $\Omega$ be the first uncountable ordinal and let $X$ be the set of all ordinals less than or equal to $\Omega$. Let $\mathscr{T}$ be the order topology and $\mathscr{B}$ the pertaining Borel-field of $X$. Then $(X, \mathscr{T})$ is a compact Hausdorff space. According to Halmos $\left[3\right.$, p. 231] the set function $v_{1} \mid \mathscr{B}$, defined by $v_{1}(B)=1$ or 0 according as $B$ does or does not contain an unbounded closed subset of $X-\Omega$, is a measure. Let $v_{2} \mid \mathscr{B}$ be the probability measure concentrated in $\Omega$. For each $T \in \mathscr{T}, v_{2}(T)=1$ implies $v_{1}(T)=1$. If $T$ is regular open and $v_{1}(T)=1$, then $\Omega \in T$, whence $v_{2}(T)=1$. Therefore $v_{1}$ and $v_{2}$ coincide for all regular open sets. However $v_{1}(X-\Omega)=1, v_{2}(X-\Omega)=0$ and $X-\Omega$ is an open set.

We remark that there exist trivial examples for measures coinciding on the regular open but not on the open sets if the topological space is normal and compact but not Hausdorff; e.g.: Let $X=\{0,1\}, \mathscr{T}=$ $\{\varnothing,\{0\}, X\}$ and $\nu_{1}, v_{2}$ be the probability measures concentrated at 0 and 1 , respectively.

COROLlaRY 3. Let $(X, \mathscr{T})$ be a perfectly normal topological space. If a sequence of measures on the Borel-field of $X$ with values in a topological group is Cauchy convergent for the regular open sets, then it is Cauchy convergent for the Borel sets, too.

Proof. A perfectly normal space is normal and every open set is a countable union of closed sets. Hence Proposition 15 of [5] implies that each measure on the Borel-field of $X$ is closed approximable. Now Theorem 1 implies the assertion.

We remark that Corollary 3 is applicable to pseudometric spaces, because a pseudometric space is perfectly normal.

\section{Auxiliary lemmata.}

LEMMA 4. Let $(X, \mathscr{T})$ be a topological space and $\mu$ be a measure on the Borel-field $\mathscr{B}$ of $X$ with values in a topological group G. Let $T_{j} \in \mathscr{T}, j \in N$, be a sequence of disjoint sets. Then for every $F \in \mathscr{F}(0)$ there exists a subsequence $N_{0} \subset N$ such that $\mu\left(\mathscr{B} \cap\left(\bigcup_{i \in N_{0}} T_{j}\right)^{*}\right) \subset F$. 
Proof. Let $N_{k} \subset N, k \in N$, be disjoint subsequences of $N$. Let $U_{k}$ : = $\bigcup_{J_{\in N_{k}}} T_{j}, k \in N$. Then $U_{k} \in \mathscr{T}, k \in N$, are disjoint whence $U_{k}^{*}=$ int $U_{k}^{c}, k \in N$, are disjoint. Hence the assertion follows from Lemma 6.

LEMMA 5. Let $(X, \mathscr{T})$ be a topological space and $\mathscr{K}$ be a system of closed sets such that

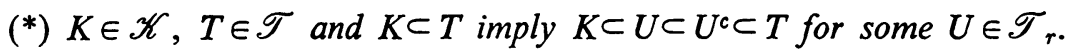
Let $\mu_{n}, n \in N$, be a sequence of $\mathscr{K}$-regular measures defined on the Borelfield $\mathscr{B}$ of $X$ with values in a topological group $G$. If $\mu_{n}, n \in N$, is Cauchy convergent on $\mathscr{T}_{r}$ then we have, for each sequence of disjoint sets $T_{j} \in \mathscr{T}$, $j \in N, \lim _{j \in N} \mu_{n}\left(T_{j}\right)=0$ uniformly in $n \in N$.

Proof. W.l.g. we may assume that $G$ is a Hausdorff group. If the assertion is wrong there exist $F_{0} \in \mathscr{F}(0)$, a sequence of disjoint sets $T_{j} \in \mathscr{T}$, $j \in N$, and a subsequence $\left(n_{j}\right)_{j \in N}$ of $N$ such that

(1) $\mu_{n_{j}}\left(T_{j}\right) \notin F_{0}$ for all $j \in N$.

Let $v_{j}:=\mu_{n_{j}}, j \in N$. There exists $F_{1} \in \mathscr{F}(0)$ with $F_{1}+F_{1} \subset F_{0}$. As $v_{j}$ is $\mathscr{K}$-regular and $\left(^{*}\right)$ is fulfilled, there exists $U_{j} \in \mathscr{T}_{\tau}$ with $U_{j} \subset U_{j}^{c} \subset T_{j}$ and

(2) $v_{j}\left(U_{j}\right) \notin F_{1}, j \in N$.

There exists a symmetric $F_{2} \in \mathscr{F}(0)$ with $F_{2}+F_{2}+F_{2}+F_{2} \subset F_{1}$. Starting with $N_{1}:=N, n_{0}:=0$ we shall show by induction over $j \in N$ that there exist sequences $N_{j+1} \subset N$ and $n_{j} \in N$ with $n_{j} \in N_{j} \supset N_{j+1}, n_{j}>n_{j-1}$, such that:

(3) $v_{n_{j}}\left(\mathscr{B} \cap\left(\bigcup_{k \in N_{j+1}} U_{k}\right)^{*}\right) \subset F_{2}$;

(4) $v_{n_{j}}\left(\bigcup_{i \in M} U_{i}\right)-v_{k}\left(\bigcup_{i \in M} U_{i}\right) \in F_{2}$ for all $k \geqq n_{j}, M \subset\left\{1, \cdots, n_{j-1}\right\}$.

Assume that the assertion is true for $j-1, j \in N$. As $\left(v_{n}\right)_{n \in N}$ is Cauchy convergent on $\mathscr{T}_{r}$, there exists $n_{j} \in N_{j}, n_{j}>n_{j-1}$, such that (4) is fulfilled. According to Lemma 4, applied to $\mu:=v_{n_{j}}$ and the sequence of disjoint sets $U_{k} \in \mathscr{T}, k \in N_{j}$, property (3) is fulfilled for some subsequence $N_{j+1} \subset N_{j}$. This proves the induction.

Now we shall show that for an appropriate regular open set $U_{0}$ the subsequence $v_{n_{j}}\left(U_{0}\right)$ of $v_{j}\left(U_{0}\right)$ is not Cauchy convergent, contradicting the assumption.

Let $N_{0}:=\{2 k: k \in N\}$ and $U_{0}:=\left(\bigcup_{k \in N_{0}} U_{n_{k}}\right)^{*} \in \mathscr{T}_{r}$. As $U_{j} \subset U_{j}^{c} \subset T_{j} \in \mathscr{T}$, $j \in N$, and $T_{j} \in \mathscr{T}, j \in N$, are disjoint, we have, for all $N^{*} \subset N$,

$$
\left(\bigcup_{j \in N^{*}} U_{j}\right)^{*}=\bigcup_{n>j \in N^{*}} U_{j}+\left(\bigcup_{n \leqq j \in N^{*}} U_{j}\right)^{*} \text { for any } n \text {. }
$$

Hence we obtain, for all $j \in N_{0}$,

$$
\begin{aligned}
v_{n_{j}}\left(U_{0}\right)-v_{n_{j-1}}\left(U_{0}\right) & \\
= & v_{n_{j}}\left(U_{n_{j}}\right)+\left(v_{n_{j}}\left(\bigcup_{j-1>k \in N_{0}} U_{n_{k}}\right)-v_{n_{j-1}}\left(\bigcup_{j-1>k \in N_{0}} U_{n_{k}}\right)\right) \\
& +v_{n_{j}}\left(\bigcup_{j<k \in N_{0}} U_{n_{k}}\right)^{*}-v_{n_{j-1}}\left(\bigcup_{j-1<k \in N_{0}} U_{n_{k}}\right)^{*} .
\end{aligned}
$$


Hence (2), (3) and (4) imply $v_{n_{j}}\left(U_{0}\right)-v_{n_{j-1}}\left(U_{0}\right) \notin F_{2}$ for all $j \in N_{0}$. As $\left(v_{j}\right)_{j \in N}$ is Cauchy convergent on $\mathscr{T}_{r}$, this yields a contradiction.

For the sake of completeness we cite the following two lemmas which are proved in [5]:

LEMMA 6. Let $\mu$ be a measure defined on a $\sigma$-ring $\mathscr{R}$ with values in a topological group $G$. If $A_{n} \in \mathscr{R}, n \in N$, are disjoint, then for every $F \in \mathscr{F}(0)$ there exists $n_{0} \in N$ such that $\mu\left(\mathscr{R} \cap \bigcup_{k \geqq n} A_{k}\right) \subset F$ for all $n \geqq n_{0}$.

Proof. Lemma 13 of [5].

LEMMA 7. Let $\mathscr{R}$ be a $\sigma$-ring and $\mathscr{T}$ be a subsystem of $\mathscr{R}$ which is closed under finite intersections and separates the subsystem $\mathscr{K}$ of $\mathscr{R}$ (i.e. for each disjoint $K_{1}, K_{2} \in \mathscr{K}$ there exist disjoint $T_{1}, T_{2} \in \mathscr{T}$ with $K_{1} \subset T_{1}$ and $K_{2} \subset T_{2}$ ). Let $G$ be a topological group and $\mu_{n}: \mathscr{R} \rightarrow G, n \in N$, a sequence of $\mathscr{K}$-regular measures such that for each sequence of disjoint sets $T_{j} \in \mathscr{T}, j \in N$ :

uniformly in $n \in N$.

$$
\lim _{j \in N} \mu_{n}\left(T_{j}\right)=0
$$

Then $\mathscr{T}$ approximates $\mathscr{K}$ from above uniformly with respect to $\mu_{n}$, $n \in N$, i.e. for all $F \in \mathscr{F}(0), K \in \mathscr{K}$, there exists $T \in \mathscr{T}, T \supset K$, such that $\mu_{n}(\mathscr{R} \cap(T-K)) \subset F$ for all $n \in N$.

Proof. Lemma 12 of [5].

ACKNOWLEDGEMENT. The author wishes to thank Mr. D. Landers for valuable suggestions.

\section{REFERENCES}

1. P. Gänssler, Compactness and sequential compactness in spaces of measures, $Z$. Wahrscheinlichkeitstheorie und Verw. Gebiete 17 (1971), 124-146.

2. - A convergence theorem for measures in regular Hausdorff spaces, Math. Scand. 29 (1971), 237-244.

3. P. R. Halmos, Measure theory, Van Nostrand, Princeton, N.J., 1950. MR 11, 504.

4. D. Landers and L. Rogge, The Hahn-Vitali-Saks and the uniformly boundedness theorem in topological groups, Manuscripta Math. 4 (1971), 351-359.

5. - Cauchy convergent sequences of regular measures with values in a topological group, Z. Wahrscheinlichkeitstheorie und Verw. Gebiete 21 (1972), 188-196.

6. B. B. Wells, Jr., Weak compactness of measures, Proc. Amer. Math. Soc. 20 (1969), 124-130. MR 38 \#6343.

Department of Mathematics, University of Cologne, Cologne, Federal REPUBLIC OF GERMANY 